\title{
MIBG scans in patients with stage 4 neuroblastoma reveal two metastatic patterns, one is associated with MYCN amplification and in MYCN-amplified tumours correlates with a better prognosis
}

\author{
Gitta Bleeker • Berthe L. van Eck-Smit • Koos H. Zwinderman • \\ Rogier Versteeg • Max M. van Noesel • Boen L. Kam • Gertjan J. Kaspers • \\ Annelies van Schie • Susan G. Kreissman • Gregory Yanik • Barbara Hero • \\ Matthias Schmidt • Geneviève Laureys • Bieke Lambert • Ingrid Øra • \\ Johannes H. Schulte • Huib N. Caron • Godelieve A. Tytgat
}

Received: 6 May 2014 / Accepted: 2 September 2014 /Published online: 30 September 2014

(C) The Author(s) 2014. This article is published with open access at Springerlink.com

\author{
Abstract \\ Purpose The aim of this study was to find clinically relevant \\ MIBG-avid metastatic patterns in patients with newly diag- \\ nosed stage 4 neuroblastoma. \\ Methods Diagnostic ${ }^{123}$ I-MIBG scans from 249 patients (123 \\ from a European and 126 from the COG cohort) were \\ Electronic supplementary material The online version of this article \\ (doi:10.1007/s00259-014-2909-1) contains supplementary material, \\ which is available to authorized users. \\ G. Bleeker • H. N. Caron • G. A. Tytgat $(\bowtie)$ \\ Department of Paediatric Oncology, Academic Medical Centre/ \\ Emma Children's Hospital, PO Box 22700, 1100 DE Amsterdam, \\ The Netherlands \\ e-mail: g.a.tytgat@amc.nl \\ G. Bleeker · R. Versteeg $\cdot$ I. Øra \\ Department of Oncogenomics, Academic Medical Centre, \\ Amsterdam, Netherlands \\ B. L. van Eck-Smit \\ Department of Nuclear Medicine, Academic Medical Centre, \\ Amsterdam, Netherlands

\section{K. H. Zwinderman} \\ Department of Biostatistics, Academic Medical Centre, Amsterdam, \\ Netherlands \\ M. M. van Noesel \\ Department of Paediatric Oncology/Haematology, Erasmus Medical \\ Centre/Sophia Children's Hospital, Rotterdam, Netherlands

\section{B. L. Kam} \\ Department of Nuclear Medicine, Erasmus Medical Centre, \\ Rotterdam, Netherlands

assessed for metastatic spread in 14 body segments and the form of the lesions: "focal" (clear margins distinguishable from adjacent background) or "diffuse" (indistinct margins, dispersed throughout the body segment). The total numbers of diffuse and focal lesions were recorded. Patients were then categorized as having lesions exclusively focal, lesions more

\section{G. J. Kaspers}

Department of Paediatric Oncology, VU University Medical Centre, Amsterdam, Netherlands

A. van Schie

Department of Nuclear Medicine, VU University Medical Centre, Amsterdam, Netherlands

\section{S. G. Kreissman}

Duke University Medical Centre, Durham, NC, USA

S. G. Kreissman • G. Yanik

Children's Oncology Group (COG), University of Florida,

Gainesville, FL, USA

G. Yanik

Department of Paediatrics, Division of Haematology and Oncology, University of Michigan, Ann Arbor, MI, USA

B. Hero

Children's Hospital, University Hospital of Cologne, Cologne, Germany

M. Schmidt

Department of Nuclear Medicine, University Hospital of Cologne,

Cologne, Germany 
focal than diffuse, lesions more diffuse than focal, or lesions exclusively diffuse.

Results Diffuse lesions affected a median of seven body segments and focal lesions a median of two body segments $(P<0.001$, both cohorts). Patients with a focal pattern had a median of 2 affected body segments and those with a diffuse pattern a median of 11 affected body segments $(P<0.001$, both cohorts). Thus, two MIBG-avid metastatic patterns emerged: "limited-focal" and "extensive-diffuse". The median numbers of affected body segments in MYCN-amplified (MNA) tumours were 5 (European cohort) and 4 (COG cohort) compared to 9 and 11, respectively, in single-copy MYCN (MYCNsc) tumours $(P<0.001)$. Patients with exclusively focal metastases were more likely to have a MNA tumour ( $60 \%$ and $70 \%$, respectively) than patients with the other types of metastases ( $23 \%$ and $28 \%$, respectively; $P<0.001)$. In a multivariate Cox regression analysis, focal metastases were associated with a better event-free and overall survival than the other types of metastases in patients with MNA tumours in the COG cohort $(P<0.01)$.

Conclusion Two metastatic patterns were found: a "limited and focal" pattern found mainly in patients with MNA neuroblastoma that correlated with prognosis, and an "extensive and diffuse" pattern found mainly in patients with MYCNsc neuroblastoma.

Keywords Neuroblastoma $\cdot$ MIBG scan $\cdot$ Metastatic patterns $\cdot$ Metastases $\cdot$ Outcome

\section{Introduction}

In about $90 \%$ of patients with neuroblastoma, ${ }^{123}$ I-MIBG scintigraphy reveals both the primary tumour and, in stage 4 neuroblastoma, especially osteomedullary metastases [1]. Two standardized methods for scoring MIBG scans have been described: the Curie method and the SIOPEN method [2-7].

\author{
G. Laureys \\ Department of Paediatric Haematology and Oncology, Ghent \\ University Hospital, Ghent, Belgium \\ B. Lambert \\ Department of Nuclear Medicine, Ghent University Hospital, Ghent, \\ Belgium \\ I. Øra \\ Department of Paediatric Oncology, Lund University Hospital, Lund, \\ Sweden \\ J. H. Schulte \\ University Children's Hospital Essen, Essen, Germany \\ H. N. Caron · G. A. Tytgat \\ Dutch Childhood Oncology Group (DCOG), The Hague, \\ Netherlands
}

These are semiquantitative and are used for assessment of tumour load and for response evaluation. However, no detailed analyses of MIBG-avid metastatic patterns in stage 4 neuroblastoma have been reported. Moreover, as well as the number of lesions (tumour load) on ${ }^{123}$ I-MIBG scans at diagnosis, osteomedullary lesions can present as focal lesions, diffuse lesions or both types. So we wondered if these would represent different types of biological lesion.

As stage 4 neuroblastoma is a heterogeneous disease with varying outcomes, we hypothesized that different disease patterns exist in patients with stage 4 neuroblastoma. Therefore, the aim of this study was to find clinically relevant MIBG-avid metastatic patterns in patients with newly diagnosed stage 4 neuroblastoma.

\section{Materials and methods}

Diagnostic ${ }^{123}$ I-MIBG scans from 249 patients with histologically proven [8] stage 4 neuroblastoma according to the International Neuroblastoma Staging System from two patient cohorts (the European cohort and the COG cohort) were included retrospectively. The European cohort comprised 123 patients from European collaborative centres diagnosed between October 1994 and September 2012 (Supplementary Table 1) and treated with different high-risk protocols: HRNBL-1/SIOPEN (26 patients) [9], GPOH NB97 (21 patients) [10], NBL HR VECI (38 patients) [11], DCOG NBL 2004/ GPOH (38 patients). The COG cohort comprised 126 patients from the COG A3973 high-risk protocol study diagnosed between March 2001 and March 2006 [12]. The European cohort was used as the study cohort, and the COG cohort as the validation cohort. Clinical patient data, available in the clinical trial databases of the centres and trial organizations were used. MYCN status was determined as specified in the GPOH, SIOPEN and COG trials (Southern blot, fluorescence in situ hybridization or array-based comparative genomic hybridization) [13-15].

For the European cohort, the institutional review board approved this retrospective study and the requirement to obtain informed consent was waived (European cohort) or written informed consent was obtained from all patients (or legal guardians) before study entry (COG cohort).

\section{${ }^{123}$ I-MIBG scans}

Diagnostic whole-body ${ }^{123}$ I-MIBG scans acquired according to protocols corresponding with European guidelines and International Neuroblastoma Risk Group taskforce recommendations were used $[1,6,16]$. Scans were excluded if metastases were non-MIBG-avid, if body parts were lacking, if the image count rate was not sufficient for adequate discrimination between body and background (inferior quality), 
if patients were receiving treatment with antihypertensive agents that antagonize the uptake of MIBG by neuroblastoma cells, and if no scan was available at diagnosis (Supplementary Table 2).

\section{Method for evaluating ${ }^{123}$ I-MIBG scans}

To be able to investigate metastatic patterns, the number of affected body segments was recorded (maximum of 14) and the form of MIBG-avid skeletal lesions was categorized as "focal" (F) or "diffuse" (D) (Fig. 1a; see Supplementary Table 3 for an example of the categorization of form). Focal metastases were hot-spots with clear margins distinguishable from the background (Fig. 1b). Diffuse metastases had no clear margins and were spread throughout the body segment (Fig. 1c). Scans were evaluated by two independent observers trained by a nuclear medicine radiologist. For the $\mathrm{COG}$ cohort the extension of metastases was also scored according to the Curie method $[2,6]$.

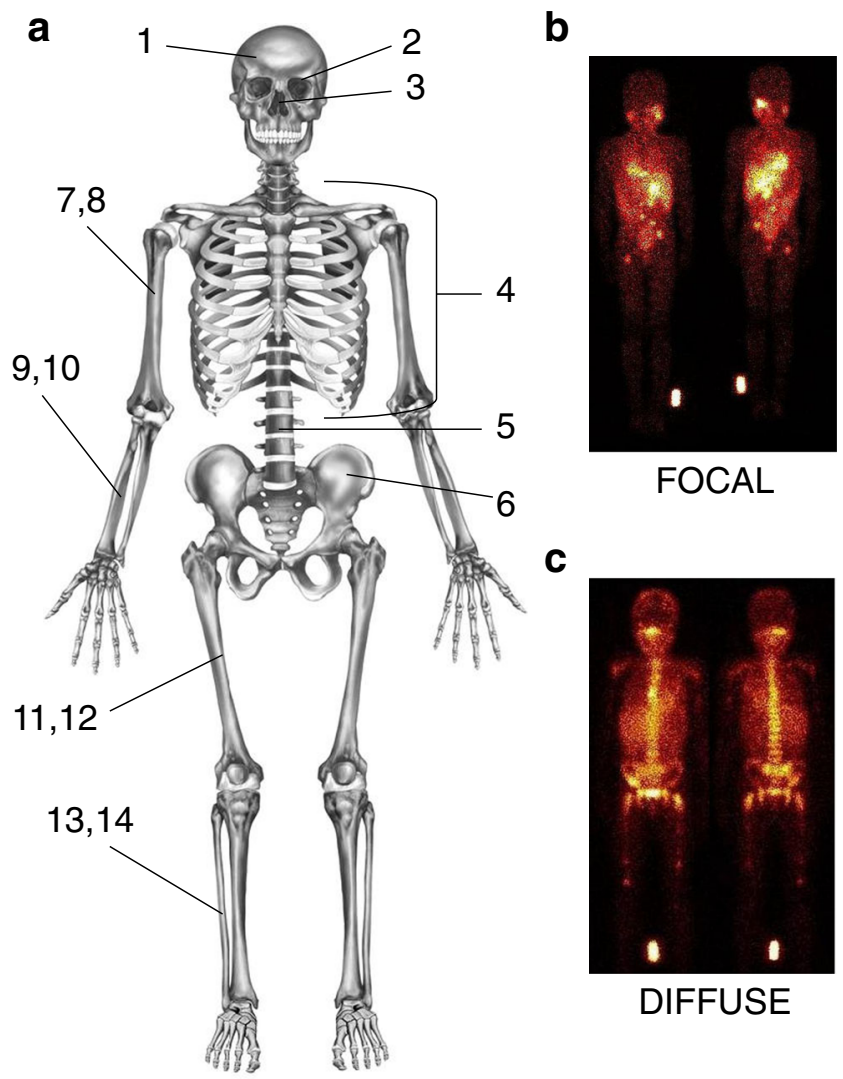

Fig. 1 Method for evaluating MIBG-avid metastatic patterns (form and number of affected body segments). a Number of affected body segments: 1 dome of skull; 2 base of skull; 3 facial bones; 4 ribs, sternum, scapula and clavicles; 5 vertebral column; 6 pelvis; 7 and 8 upper arms (left and right); 9 and 10 forearms and hands (left and right); 11 and 12 upper legs (left and right); 13 and 14: lower legs and feet (left and right). b, c Lesion form: focal (b) lesions imaged as hot-spots with clear margins, distinguishable from the background; diffuse (c) lesions without clear margins and uptake spread throughout the body segment
Statistical analysis

Interobserver variability was quantified using the kappa coefficient before discordant findings were resolved by consensus. All reported correlations and outcome analyses were performed on the consensus scores. Correlations were tested for statistical significance using the Mann-Whitney $U$ test, the Kruskal Wallis test or Fisher's Exact test. Survival was analysed using the Kaplan-Meier life-table method for the COG cohort because this cohort was homogeneously treated over a fixed time period. The median follow-up was 6.1 years (1.3 to 9.5 years). Event-free survival (EFS) was calculated as the time from diagnosis to the first event (relapse, progression, death) or last examination if no event occurred. Overall survival (OS) was calculated as the time from diagnosis to death or last examination. Differences in OS and EFS between patients with different metastatic patterns were analysed using the log-rank test. A multivariate Cox regression analysis of the form of metastases, age, and Curie score, stratified by MYCN status, was also performed. Therefore metastases were divided into two categories (dichotomized) according to form (exclusively focal versus the others, i.e. focal $\geq$ diffuse + diffuse $>$ focal+diffuse), and the patients were divided according to the cut-off age of 18 months [17] and according to the median Curie score of 12 .

To test the effect of tumour burden at diagnosis on outcome, the Curie score was determined in the COG cohort, and outcome was evaluated in term of both previously published cut-off scores of 2 and $9[3,7]$ and also a cut-off score of 12 (median score in the COG cohort).

\section{Results}

Patient characteristics

We included 123 out of 149 of the available diagnostic ${ }^{123} \mathrm{I}-$ MIBG scans from patients of the European cohort. In the COG A3973 high-risk cohort, in 306 patients, 91 diagnostic scans were ${ }^{131} \mathrm{I}-\mathrm{MIBG}$ scans. Clinical and biological characteristics of 170 of 215 patients with ${ }^{123}$ I-MIBG scans were available and evaluated [12]. Of these $170{ }^{123} \mathrm{I}$-MIBG scans, 126 were included. Excluded scans are described in Supplementary Table 2 for the two cohorts: non-MIBG-avid metastases in 21 and 20 patients, inferior quality in 2 and 23, use of antihypertensive agents in 2 and 0 , and treatment before the scanning procedure in 1 and 1 patient. The distribution of age and MYCN status was comparable in both cohorts (Table 1), except exclusion of patients younger than 12 months with single-copy MYCN (MYCNsc) neuroblastoma in the high-risk COG cohort. The characteristics of the excluded patients were comparable in the two cohorts. 
Table 1 Patient characteristics

\begin{tabular}{llll}
\hline MYCN status & Age & $\begin{array}{l}\text { European } \\
\text { cohort }(n=123)^{\mathrm{a}}\end{array}$ & $\begin{array}{l}\text { COG cohort } \\
(n=126)^{\mathrm{b}}\end{array}$ \\
\hline Amplified $^{\mathrm{c}}$ & $<12$ months & 3 & 2 \\
& $12-18$ months & 7 & 9 \\
& 18 months to 12 years & 28 & 34 \\
& $\geq 12$ years & 0 & 0 \\
& Unknown & 1 & 0 \\
Single copy & Total & 39 & 45 \\
& $<12$ months & 16 & 0 \\
& $12-18$ months & 6 & 4 \\
& 18 months to 12 years & 54 & 71 \\
& $\geq 12$ years & 2 & 5 \\
& Unknown & 0 & 0 \\
No data & Total & 78 & 80 \\
& $<12$ months & 0 & 0 \\
& $12-18$ months & 1 & 0 \\
& 18 months to 12 years & 5 & 0 \\
& $\geq 12$ years & 0 & 0 \\
& Unknown & 0 & 1 \\
& Total & 6 & 1 \\
& & &
\end{tabular}

${ }^{a}$ Median age 2.7 years (range $0-16.5$ years)

${ }^{\mathrm{b}}$ Median age 2.9 years (range $0.8-15.2$ years)

${ }^{\mathrm{c}} \mathrm{MYCN}$ amplification was considered present if eight or more copies were detected [10-12]

Focal and diffuse lesions

A total 966 lesions were identified in the European cohort (123 patients; Table 2) affecting 928 body segments. Of these lesions, 292 (30\%) were focal and 674 (70\%) were diffuse. Representative ${ }^{123}$ I-MIBG scans are shown in Fig. 1b, c. The 292 focal lesions were present in 101 of 123 patients, with a median of 2 per patient compared to 674 diffuse lesions in 95 of 123 patients with a median of $7(P<0.0001$; Table 2$)$. In the COG cohort A total of 984 lesions were identified in the COG cohort (123 patients) affecting 969 body segments. Of these lesions, $264(27 \%)$ in 95 patients were focal and $720(73 \%)$ in 103 patients were diffuse. Diffuse lesions affected a median of 7 body segments per patient and focal lesions a median of 2 body segments per patient $(P<0.0001$; Table 2$)$.
"Limited-focal" and "extensive-diffuse" MIBG-avid metastatic patterns

Patients present with only focal, only diffuse, or both forms of metastases. The numbers of patients with "exclusively focal" (focal), "focal more than/equal to diffuse" (focal $\geq$ diffuse), "more diffuse than focal" (diffuse $>$ focal), and "exclusively diffuse" (diffuse) lesions were recorded. The distributions of the patterns were very similar in the European and COG cohorts, with a slight preponderance of the diffuse $>$ focal pattern (38\% and $39 \%$ ), with $23 \%$ and $18 \%$ focal, $21 \%$ and $18 \%$ focal $\geq$ diffuse, and $18 \%$ and $25 \%$ diffuse, respectively (Fig. 2a, b).

The association between the form and the number of affected body segments were then analysed for each patient. In the European cohort, focal, focal $\geq$ diffuse, diffuse $>$ focal and diffuse lesions were identified in a median of 2, 6, 11 and 11 body segments, respectively (Fig. 2c). There were significant differences between the form groups in relation to the number of affected body segments: focal versus diffuse, focal $\geq$ diffuse versus diffuse $>$ focal and focal versus the three other groups, all $P<0.001$. In the COG cohort these patterns were very similar and significant relationships between the form of metastases and the number of affected body segments were also seen (Fig. 2d). The actual numbers of focal and diffuse lesions in each individual patient are shown in Supplementary Table 4.

\section{"Limited-focal" patterns associated with MYCN} amplification

The differences in MIBG-avid metastatic patterns suggest a biological difference between the MYCN status groups. We therefore investigated whether the $\mathrm{MYCN}$ gene could be involved. MYCN status was clearly associated with the number of affected body segments (Fig. 3) as well as with the form of metastases (Table 3). Patients with MYCN-amplified (MNA) tumours had significantly fewer affected body segments. The median numbers of affected body segments in the European and COG patients with MNA tumours were 5 and 4, respectively, and in the European and COG patients with MYCNsc tumours were 9 and 11, respectively $(P<0.001$ for
Table 2 Characteristics of metastatic lesions

\begin{tabular}{llllll}
\hline & & Focal & Diffuse & Total & $P$ value \\
\hline European cohort & Number of lesions & $292(30 \%)$ & $674(70 \%)$ & 966 & \\
& Number of patients & 101 & 95 & 123 & \\
& Lesions per patient, median (range) & $2(1-9)$ & $7(1-14)$ & & $<0.001$ \\
COG cohort & Number of lesions & $264(27 \%)$ & $720(73 \%)$ & 984 & \\
& Number of patients & 95 & 103 & 126 & \\
& Lesions per patient, median (range) & $2(1-8)$ & $7(1-14)$ & & $<0.001$ \\
\hline
\end{tabular}


Fig. 2 Forms of metastases and numbers of affected body segments per patient. a, b Distribution of forms of metastases in the European cohort (a) and the COG cohort (b). The numbers of patients with focal, diffuse and both types of lesions are shown. Patients were categorized as exclusively focal $(F)$, exclusively diffuse $(D)$, focal $\geq$ diffuse $(F \geq D)$ or diffuse $>$ focal $(D>F)$. c, d Relationship between form of metastases and number of affected body segments per individual patient in the European cohort (c) and the COG cohort (d). Each data point represents a patient; the horizontal lines indicate the median number of affected body segments
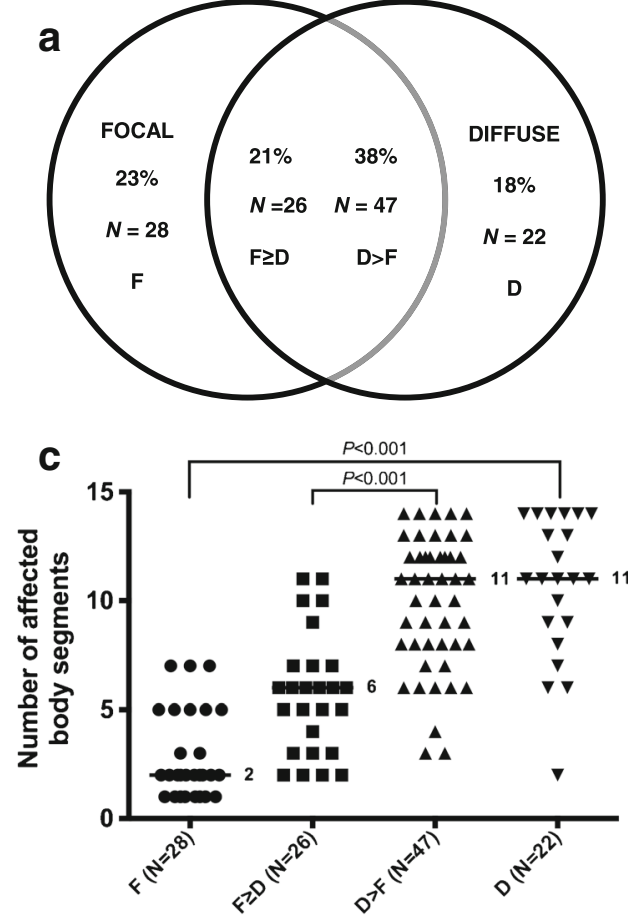

Form of metastases
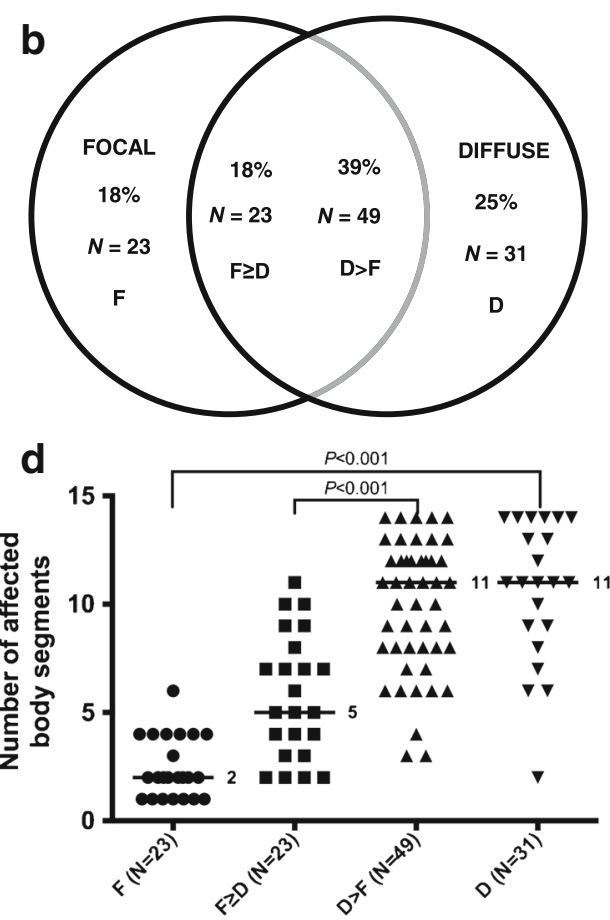

Form of metastases
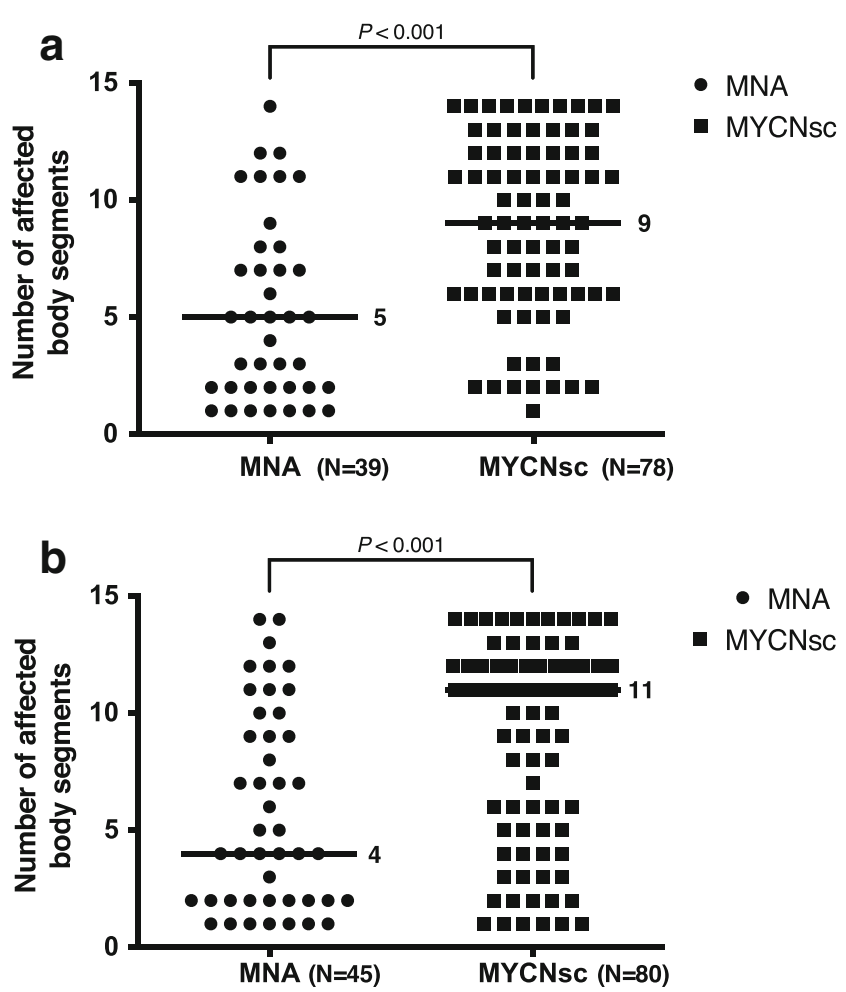

Fig. 3 Relationship between MYCN status and number of affected body segments per patient: a European cohort ( $n=123$; six patients no data on MYCN status). b COG cohort ( $n=126$; one patient no data on MYCN status). Each data point represents a patient; horizontal lines median numbers of affected body segments. MNA MYCN amplification, MYCNsc single-copy MYCN both cohorts; Fig. 3). MNA tumour was found in $67 \%$ of patients (18/27) with focal lesions in the European cohort and in $70 \%$ of such patients (16/23) in the COG cohort (Table 3 ). In contrast, MNA tumour was found in only $23 \%$ of patients $(21 / 90)$ in the other metastatic groups (focal $\geq$ diffuse + diffuse $>$ focal + diffuse; $P<0.001)$ in the European cohort and in $28 \%$ of such patients $(29 / 102 ; P<0.001)$ in the COG cohort.

Prognostic value of MIBG-avid metastatic patterns

Next, we investigated the prognostic impact of the identified MIBG-avid metastatic patterns. This analysis was only performed in the COG cohort. The European cohort received more variable treatment due to the longer inclusion period, which might have had an impact on OS and EFS, but not on the metastatic pattern as these were investigated at diagnosis only.

COG patients with exclusively focal metastases had a small but statistically significant better OS, but not EFS, than patients in the other metastatic groups $(95 \%$ confidence intervals, CI: $52 \pm 20 \%$ vs. $35 \pm 9 \%$ for 5 -year EFS, $P=0.191 ; 73 \pm$ $19 \%$ vs. $49 \pm 10 \%$ for 5-year OS, $P=0.050$; Supplementary Fig. 1). Among patients with MNA tumours, those with focal lesions had a much better EFS and OS than those in the other metastatic groups (95\% CI: $63 \pm 24 \%$ vs. $21 \pm 15 \%$ for 5 -year EFS, $P=0.006 ; 81 \pm 20 \%$ vs. $28 \pm 17 \%$ for 5 -year OS, $P=$ 0.001 ; Fig. $4 \mathrm{a}, \mathrm{b}$ ). Among patients with MYCNsc tumours, no 
Table 3 MYCN status in relation to the form of metastases

\begin{tabular}{|c|c|c|c|c|c|c|}
\hline \multirow[t]{3}{*}{ MYCN status } & \multicolumn{3}{|c|}{ European cohort } & \multicolumn{3}{|c|}{ COG cohort } \\
\hline & \multicolumn{2}{|l|}{ Form } & \multirow[t]{2}{*}{ Total } & \multicolumn{2}{|l|}{ Form } & \multirow[t]{2}{*}{ Total } \\
\hline & Focal & $\begin{array}{l}\text { All other } \\
\text { groups }^{\text {a }}\end{array}$ & & Focal & $\begin{array}{l}\text { All other } \\
\text { groups }^{\mathrm{a}}\end{array}$ & \\
\hline Amplified & 18 & 21 & 39 & 16 & 29 & 45 \\
\hline Single copy & 9 & 69 & 78 & 7 & 73 & 80 \\
\hline Total & 27 & 90 & 117 & 23 & 102 & 125 \\
\hline$P$ value & & & $<0.001$ & & & $<0.001$ \\
\hline
\end{tabular}

${ }^{\mathrm{a}}$ Focal $\geq$ diffuse + diffuse $>$ focal + diffuse

significant nor large differences in EFS and OS were found between those with focal lesions and those in all other metastatic groups (95 \% CI: $29 \pm 34 \%$ vs. $41 \pm$ $12 \%$ for 5 -year EFS, $P=0.401 ; 57 \pm 37 \%$ vs. $58 \pm 12 \%$ for 5-year OS, $P=0.930$; Fig. 4c, d).

In the separate multivariate Cox regression analyses in patients with MNA tumour and those with MYCNsc tumours the form of metastases in MNA neuroblastomas showed independent prognostic value in the presence of potential prognostic factors (age and Curie score at diagnosis; Table 4). Using the Curie score as a dichotomized
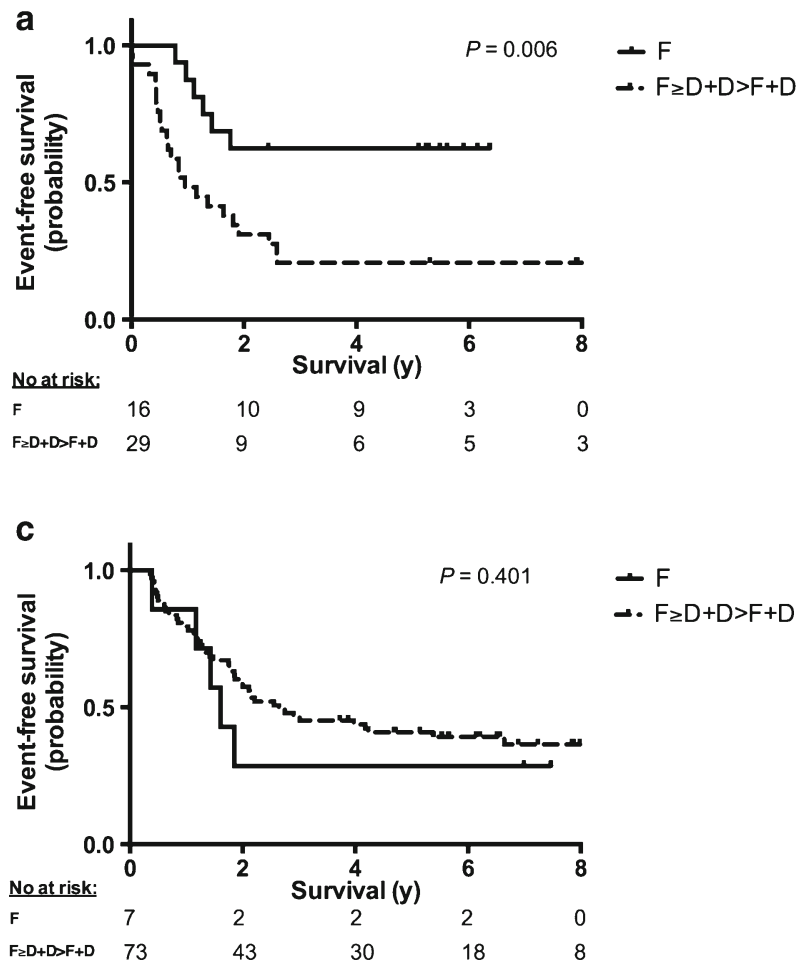

Fig. 4 Event-free survival (EFS) and overall survival (OS) according to the form of metastases stratified by MYCN status (a, b MYCN-amplified tumours; $\mathbf{c}, \mathbf{d}$ single-copy MYCN tumours). EFS $(\mathbf{a}, \mathbf{c})$ and OS $(\mathbf{b}, \mathbf{d})$ in patients with focal lesions $(F)$ versus those with all other types of lesions variable with previously published cut-off scores of 2 and $9[3,7]$ and also with a cut-off score of 12 (median score in the COG cohort), did not result in a significant prediction of outcome in either the univariate or the multivariate analyses. So correcting for the Curie score did not have impact on outcome.

We conclude that in patients with MNA tumours, patients with exclusively focal metastases have a significantly better outcome than patients with (additional) diffuse metastases.

\section{Interobserver variability}

The interobserver variability in evaluating the ${ }^{123} \mathrm{I}-\mathrm{MIBG}$ scans was "moderate" to "almost perfect", with median $\mathrm{K}$ values for affected body segments and for form of metastases per body segment of $0.9(0.7-1.0)$ and $0.5(0.3-1.0)$, respectively in the European cohort, and of $0.9(0.7-0.9)$ and 0.6 (0.3-0.8), respectively, in the COG cohort (for details see Supplementary Table 5). The $k$ value for the form of metastases on a patient basis categorized as focal vs. focal $\geq$ diffuse + diffuse $>$ focal + diffuse was $0.7(P<0.001)$ with $89 \%$ concordant findings. All discordant findings were resolved by consensus. Performing all analyses again with single observer rates did not change the findings.
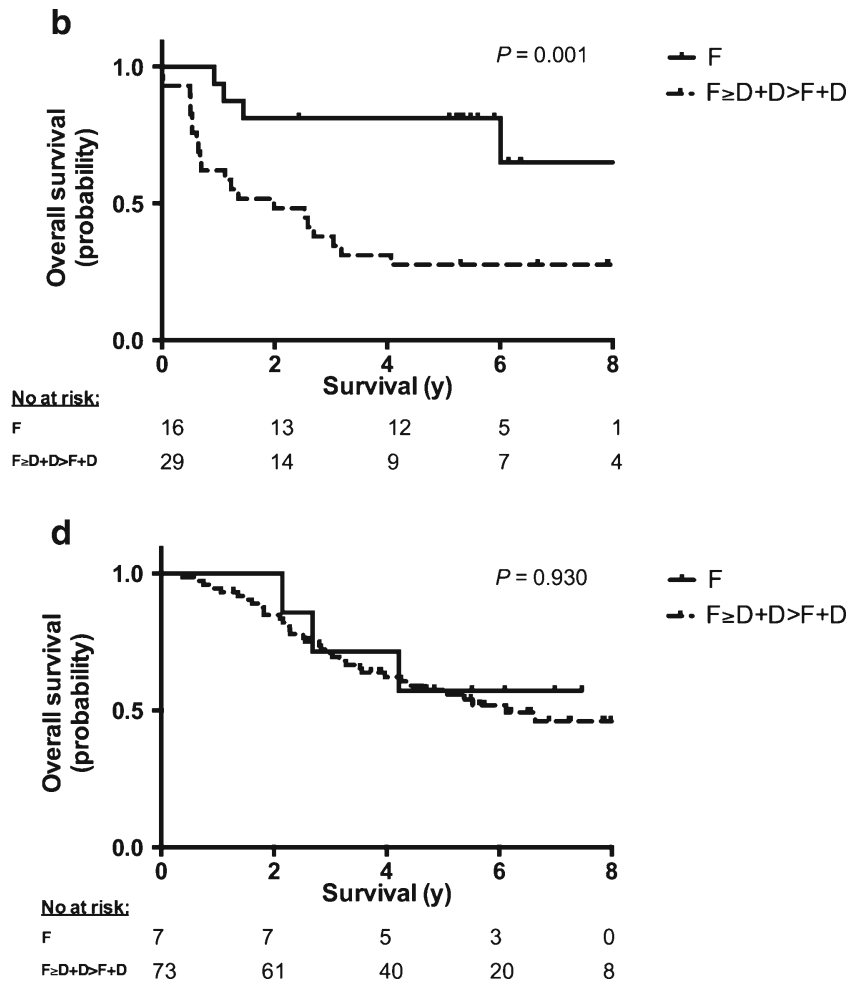

(focal $\geq$ diffuse + diffuse $>$ focal + diffuse, $F \geq D+D>F+D$ ). (No at risk number of patients at risk, i.e. still alive, at the corresponding time points, $y$ years after diagnosis) 
Table 4 Multivariate Cox regression analysis of survival in the COG cohort stratified by MYCN status

\begin{tabular}{|c|c|c|c|c|c|c|c|}
\hline \multirow[t]{2}{*}{ MYCN status } & \multirow[t]{2}{*}{ Variable } & \multicolumn{3}{|c|}{ 5-year event-free survival } & \multicolumn{3}{|c|}{ 5-year overall survival } \\
\hline & & Hazard ratio & $95 \% \mathrm{CI}$ & $P$ value & Hazard ratio & $95 \% \mathrm{CI}$ & $P$ value \\
\hline \multirow[t]{3}{*}{ Amplified } & Age ( $<18$ months vs. $\geq 18$ months $)$ & 1.3 & $0.6-3.0$ & 0.540 & 1.4 & $0.6-3.5$ & 0.472 \\
\hline & Curie score $(<12$ vs. $\geq 12)$ & 1.2 & $0.5-2.9$ & 0.607 & 1.2 & $0.5-2.9$ & 0.679 \\
\hline & Form of metastases (focal vs. all other forms ${ }^{\mathrm{a}}$ ) & 0.3 & $0.1-0.7$ & 0.010 & 0.2 & $0.04-0.6$ & 0.005 \\
\hline \multirow[t]{3}{*}{ Single copy } & Age ( $<18$ months vs. $\geq 18$ months $)$ & 0.9 & $0.2-3.7$ & 0.860 & 0.5 & $0.07-3.8$ & 0.497 \\
\hline & Curie score $(<12$ vs. $\geq 12)$ & 0.7 & $0.3-1.4$ & 0.291 & 0.9 & $0.4-1.9$ & 0.697 \\
\hline & Form of metastases (focal vs. all other forms ${ }^{\mathrm{a}}$ ) & 1.9 & $0.7-5.6$ & 0.230 & 1.1 & $0.3-4.3$ & 0.848 \\
\hline
\end{tabular}

${ }^{\mathrm{a}}$ Focal $\geq$ diffuse + diffuse $>$ focal + diffuse

\section{Discussion}

This study identified two MIBG-avid metastatic patterns in patients with newly diagnosed stage 4 neuroblastoma: a "limited and focal" pattern found mainly in patients with MNA neuroblastoma, and an "extensive and diffuse" pattern found mainly in patients with MYCNsc neuroblastoma. The obvious difference between focal and diffuse metastases is still not understood. In international guidelines skeletal uptake of MIBG is reported to be visible as focal or diffuse lesions [18], and the SIOPEN scoring method distinguishes between discrete foci and diffuse lesions [6]. In the literature, "focal" lesions are mostly reported as bone metastases $[19,20]$ and "diffuse" lesions as bone marrow metastases [19, 21-24]. As almost $95 \%$ of patients with stage 4 neuroblastoma have bone marrow involvement at diagnosis, the nature of focal and diffuse lesions was not studied in our cohort. This needs to be done in a prospective study, comparing MIBG scintigraphy, immunocytological bone marrow involvement and MRI for anatomical localization of the lesions.

To our knowledge, no classification of stage 4 tumours based on MIBG-avid metastatic patterns has been described. The recognition of two different metastatic patterns in stage 4 neuroblastoma suggests that multiple and different underlying molecular alterations might be involved in the process of metastases. Indeed the focal pattern was significantly associated with amplification of MYCN, which is considered a major tumour-driving gene. Furthermore, only in patients with MNA tumours, focal metastases were significantly associated with a more favourable outcome than diffuse metastases, and in this subset of patients different biological processes might be identified. Why this phenomenon was not seen in patients with MYCNsc tumours needs to be studied further.

A major question arising from this study is whether MYCN activity is responsible for the focal growth of metastatic lesions, and whether and how it relates to the fewer affected body segments. Not all patients with predominantly focal lesions had MNA tumours. However, in another study in our laboratory a subset of MYCNsc neuroblastomas were found to have high MYCN protein expression. We hypothesize that the patients with focal lesions with MYCNsc tumours might have had this MYCN expression profile [25]. The intriguing results of this study require substantial biological research, e.g. association with gene expression and molecular data, and in vitro and in vivo MYCN manipulation studies, to elucidate the functional role for MYCN in the metastatic spread of neuroblastoma cells. MYCN expression has been reported to correlate with a lower norepinephrine transporter (NET) protein expression, and in turn a lower NET protein expression was correlated with low MIBG avidity [26]. As we found that patients with MNA neuroblastoma had not only a predominantly focal form of metastases but also significantly fewer involved body segments on MIBG imaging at diagnosis, we hypothesize that the lower NET expression in MNA tumours causes the focal pattern.

Although two well-performing methods for scoring MIBG scans are used internationally, we developed our own method of evaluation as a tool to find MIBG-avid metastatic patterns with a qualitative variable (the form of metastases per body segment) as the key variable [2, 3, 6] (Supplementary Table 6). The novelty of our findings is that the form of metastases at diagnosis, an aspect that is not scored with the Curie method, was significantly correlated with outcome in the MNA group. If a prospective study in a homogeneously treated cohort can confirm the prognostic relevance of the metastatic pattern in patients with stage 4 neuroblastoma, eventually patients with stage 4 neuroblastoma might be subdivided in two risk groups. In addition, in future it might then be possible to treat patients with different metastatic patterns according to different treatment protocols that are more targeted at their biological background.

Although the distinction between focal and diffuse metastases was not always very clear, especially because sometimes the two forms of metastases were present in one body segment, all discordant findings could be resolved by consensus. Furthermore, the results of our correlation and prognostic analyses were comparable when using single observer scores. Since the study cohort consisted of ${ }^{123}$ I-MIBG scans obtained 
over a long period of time, scan quality might have been heterogeneous and not equally distributed between the subgroups. Therefore our results should be confirmed in a cohort of patients scanned according to a uniform protocol.

In this study we included only ${ }^{123} \mathrm{I}-\mathrm{MIBG}$ scans, but since Naranjo et al. reported no difference in outcome between scoring of ${ }^{123}$ I-MIBG and ${ }^{131}$ I-MIBG scans [12], it is debatable whether ${ }^{131}$ I-MIBG scans should have been excluded. However, this project was started many years ago and at that time ${ }^{123}$ I-MIBG scans were reported to be of better quality. In the Cox regression analysis, we included only the COG cohort, a homogeneous patient population treated according to one protocol (COG A3973 protocol). The European cohort was a heterogeneous cohort because these patients had been treated according to different protocols, and therefore this was not an ideal cohort for studying survival. A limitation of the COG cohort was that not all patients had a follow-up of 5 years. A prospective study including patients from the same treatment protocol with only digital diagnostic ${ }^{123}$ I-MIBG scans performed according to the same scanning procedures and evaluated by central review might resolve these problems.

In conclusion, our study clearly showed the existence of two relevant MIBG-avid metastatic patterns in newly diagnosed neuroblastoma: an "extensive and diffuse" MIBG-avid metastatic pattern found mainly in patients with MYCNsc tumours, and a "limited and focal" pattern found mainly in patients with MNA tumours. In patients with MNA tumours, focal metastases had a better prognosis than the other types of metastases. These two patterns most likely reflect different biological processes that should be explored further with the aim of providing a better understanding of the heterogeneous behaviour of high-risk tumours.

Acknowledgments We would like to thank Fran Laurie and staff at the Quality Assurance Review Centre (QARC) for their tremendous support for this project.

\section{Conflicts of interest None.}

Grant support This work was supported by Kika (Children Cancer Free Foundation) and Tom Voûte Foundation.

Open Access This article is distributed under the terms of the Creative Commons Attribution License which permits any use, distribution, and reproduction in any medium, provided the original author(s) and the source are credited.

\section{References}

1. Boubaker A, Bischof DA. MIBG scintigraphy for the diagnosis and follow-up of children with neuroblastoma. Q J Nucl Med Mol Imaging. 2008;52(4):388-402.

2. Ady N, Zucker JM, Asselain B, Edeline V, Bonnin F, Michon J, et al. A new 123I-MIBG whole body scan scoring method - application to the prediction of the response of metastases to induction chemotherapy in stage IV neuroblastoma. Eur J Cancer. 1995;31A(2):256-61.

3. Decarolis B, Schneider C, Hero B, Simon T, Volland R, Roels F, et al. Iodine-123 metaiodobenzylguanidine scintigraphy scoring allows prediction of outcome in patients with stage 4 neuroblastoma: results of the Cologne interscore comparison study. J Clin Oncol. 2013;31: 944-51.

4. Katzenstein HM, Cohn SL, Shore RM, Bardo DM, Haut PR, Olszewski $\mathrm{M}$, et al. Scintigraphic response by 123I-metaiodobenzylguanidine scan correlates with event-free survival in high-risk neuroblastoma. J Clin Oncol. 2004;22(19):3909-15.

5. Matthay KK, Edeline V, Lumbroso J, Tanguy ML, Asselain B, Zucker JM, et al. Correlation of early metastatic response by 123Imetaiodobenzylguanidine scintigraphy with overall response and event-free survival in stage IV neuroblastoma. J Clin Oncol. 2003;21(13):2486-91.

6. Matthay KK, Shulkin B, Ladenstein R, Michon J, Giammarile F, Lewington V, et al. Criteria for evaluation of disease extent by (123)Imetaiodobenzylguanidine scans in neuroblastoma: a report for the International Neuroblastoma Risk Group (INRG) Task Force. Br J Cancer. 2010;102(9):1319-26.

7. Yanik GA, Parisi MT, Shulkin BL, Naranjo A, Kreissman SG, London WB, et al. Semiquantitative $\mathrm{mIBG}$ scoring as a prognostic indicator in patients with stage 4 neuroblastoma: a report from the Children's Oncology Group. J Nucl Med. 2013;54:541-8.

8. Brodeur GM, Pritchard J, Berthold F, Carlsen NL, Castel V, Castelberry RP, et al. Revisions of the international criteria for neuroblastoma diagnosis, staging, and response to treatment. J Clin Oncol. 1993;11(8):1466-77.

9. Veal GJ, Nguyen L, Paci A, Riggi M, Amiel M, Valteau-Couanet D, et al. Busulfan pharmacokinetics following intravenous and oral dosing regimens in children receiving high-dose myeloablative chemotherapy for high-risk neuroblastoma as part of the HR-NBL-1/ SIOPEN trial. Eur J Cancer. 2012;48(16):3063-72.

10. Berthold F, Hero B, Kremens B, Handgretinger R, Henze G, Schilling FH, et al. Long-term results and risk profiles of patients in five consecutive trials (1979-1997) in stage 4 neuroblastoma over 1 year of age. Cancer Lett. 2003;197:11-7.

11. de Kraker J, Hoefnagel KA, Verschuur AC, van Eck B, van Santen $\mathrm{HM}$, Caron HN. Iodine-131-metaiodobenzylguanidine as initial induction therapy in stage 4 neuroblastoma patients over 1 year of age. Eur J Cancer. 2008;44(4):551-6.

12. Naranjo A, Parisi MT, Shulkin BL, London WB, Matthay KK, Kreissman SG, et al. Comparison of 123I-metaiodobenzylguanidine (MIBG) and 131I-MIBG semi-quantitative scores in predicting survival in patients with stage 4 neuroblastoma: a report from the Children's Oncology Group. Pediatr Blood Cancer. 2011;56(7): 1041-5.

13. Ambros IM, Benard J, Boavida M, Bown N, Caron H, Combaret V, et al. Quality assessment of genetic markers used for therapy stratification. J Clin Oncol. 2003;21(11):2077-84.

14. Ambros PF, Ambros IM. Pathology and biology guidelines for resectable and unresectable neuroblastic tumors and bone marrow examination guidelines. Med Pediatr Oncol. 2001;37(6): $492-504$.

15. Ambros PF, Ambros IM, Brodeur GM, Haber M, Khan J, Nakagawara A, et al. International consensus for neuroblastoma molecular diagnostics: report from the International Neuroblastoma Risk Group (INRG) Biology Committee. Br J Cancer. 2009;100(9): 1471-82.

16. Bombardieri E, Giammarile F, Aktolun C, Baum RP, Bischof Delaloye A, Maffioli L, et al. 131I/123I-metaiodobenzylguanidine (mIBG) scintigraphy: procedure guidelines for tumour imaging. Eur J Nucl Med Mol Imaging. 2010;37(12):2436-46.

17. Cohn SL, Pearson AD, London WB, Monclair T, Ambros PF, Brodeur GM, et al. The International Neuroblastoma Risk Group 
(INRG) classification system: an INRG Task Force report. J Clin Oncol. 2009;27(2):289-97.

18. Olivier P, Colarinha P, Fettich J, Fischer S, Frökier J, Giammarile F, et al. Guidelines for radioiodinated MIBG scintigraphy in children. Eur J Nucl Med Mol Imaging. 2003;30(5):B45-50.

19. Hadj-Djilani NL, Lebtahi NE, Delaloye AB, Laurini R, Beck D. Diagnosis and follow-up of neuroblastoma by means of iodine-123 metaiodobenzylguanidine scintigraphy and bone scan, and the influence of histology. Eur J Nucl Med. 1995;22(4):322-9.

20. Osmanagaoglu K, Lippens M, Benoit Y, Obrie E, Schelstraete K, Simons M. A comparison of iodine-123 meta-iodobenzylguanidine scintigraphy and single bone marrow aspiration biopsy in the diagnosis and follow-up of 26 children with neuroblastoma. Eur J Nucl Med. 1993;20(12):1154-60.

21. Claudiani F, Stimamiglio P, Bertolazzi L, Cabria M, Conte M, Villavecchia GP, et al. Radioiodinated meta-iodobenzylguanidine in the diagnosis of childhood neuroblastoma. Q J Nucl Med. 1995;39(4 Suppl 1):21-4.
22. Giammarile F, Olier AL, Lumbroso J. Diffuse bone marrow uptake [123I]MIBG in neuroblastoma: an "MIBG super scan" case report. Q J Nucl Med. 1995;39(2):119-21.

23. Lebtahi N, Gudinchet F, Nenadov-Beck M, Beck D, Bischof DA. Evaluating bone marrow metastasis of neuroblastoma with iodine-123-MIBG scintigraphy and MRI. J Nucl Med. 1997;38(9):1389-92.

24. Shah Syed GM, Naseer H, Usmani GN, Cheema MA. Role of iodine131 MIBG scanning in the management of paediatric patients with neuroblastoma. Med Princ Pract. 2004;13(4):196-200.

25. Valentijn LJ, Koster J, Haneveld F, Aissa RA, van Sluis P, Broekmans ME, et al. Functional MYCN signature predicts outcome of neuroblastoma irrespective of MYCN amplification. Proc Natl Acad Sci U S A. 2012;109(47):19190-5.

26. DuBois SG, Geier E, Batra V, Yee SW, Neuhaus J, Segal M, et al Evaluation of norepinephrine transporter expression and metaiodobenzylguanidine avidity in neuroblastoma: a report from the Children's Oncology Group. Int J Mol Imaging. 2012;2012:250834. 